\title{
COMPORTAMIENTO DE LA ESCORRENTÍA SUPERFICIAL EN LAS VERTIENTES DE UNA CUENCA HIDROGRÁFICA EN EL ECOSISTEMA DEHESA. CUENCA DE GUADALPERALÓN (PROVINCIA DE CÁCERES)
}

\author{
Antonio CEBALLOS y Susanne SCHNABEL \\ Departamento de Geografía y Ordenación del Territorio \\ Universidad de Extremadura
}

\begin{abstract}
Resumen: En una pequeña cuenca bajo explotación de dehesa se investiga la producción de escorrentía superficial en vertientes. Los resultados obtenidos muestran que la determinación del umbral de escorrentía es compleja y que los factores que mejor explican la generación y producción de escorrentía en las vertientes son la precipitación total y la intensidad máxima en 10 minutos.

Palabras clave: ecosistema dehesa, escorrentía superficial, vertientes.

Abstract: In a small catchment under the dehesa landuse system overland flow production on slopes is investigated. The obtained results show that the determination of the threshold value for the generation of runoff is complex. Total precipitation and maximum intensity in 10 minutes are the principal factors that explain the production and generation of slope runoff.
\end{abstract}

Key words: dehesa ecosystem, overland flow, slopes.

\section{INTRODUCCIÓN}

Desde 1990 se están estudiando los factores y los procesos hidrológicos y erosivos de las zonas de dehesa en una cuenca experimental en Extremadura (GÓMEZ AMELIA y SCHNABEL, 1992). Se ha investigado la erosión laminar en vertientes, erosión en cárcava, el factor vegetación en los procesos erosivos (BERNET et al., 1994; SCHNABEL y GOMEZ AMELIA, 1993; SCHNABEL et al., 1996; SCHNABEL, 1997) y el comportamiento hidrológico de la cuenca (CEBALLOS, 1997; CEBALLOS et al., 1996; CEBALLOS y SCHNABEL, en prensa). El ambiente estudiado corresponde al de una dehesa bajo explotación ganadera, donde la poda y extracción de leña se realizan esporádicamente y el pastoreo es constante. 
La investigación que se está llevando a cabo tiene un gran interés ecológico por e 1 elevado valor paisajístico y ambiental de las dehesas, así como por su importancia económica, ya que de la sostenibilidad de estas explotaciones depende la supervivencia de gran parte de la población de la zona. Esta situación es representativa del oeste-suroeste del territorio español, donde la dehesa constituye el $52 \%$ de la superficie agrícola (CAMPOS PALACÍN, 1993). A pesar de su importancia espacial, económica y ecológica, no hay información sobre su dinámica hidrológica y erosiva.

El objetivo principal del presente trabajo es el estudio de los factores principales que intervienen en la generación de escorrentía superficial en las vertientes de la cuenca y la determinación de su grado de influencia. Se entiende por flujo o escorrentía superficial la parte de la escorrentía que viaja sobre la superficie de la tierra antes de llegar a conectar con una corriente o canal por medio del cual alcanzar la salida de la cuenca (FREEZE, 1972; GERITS et al., 1990).



Figura 1.- Diagrama triangular que muestra el origen de diversos tipos de flujo en relación con las características de la cuenca (ANDERSON y BURT, 1990).

Se han utilizado los datos correspondientes a dos años hidrológicos completos (1994-95 y 1995-96) con un comportamiento pluviométrico muy contrastado. 
Tradicionalmente se asocian diferentes tipos de flujo a determinadas situaciones en donde confluyen una serie de factores característicos: ambientes áridos y semiáridos, con suelos degradados, favorecen el desarrollo del flujo superficial "hortoniano", mientras que suelos forestales en zonas de lluvias poco intensas (ambientes húmecio y subhúmedo) facilitan la articulación de flujo superficial de saturación y zonas cóncavas y de convergencia de flujo provocan flujo subsuperficial y de retorno (Fig. 1). No obstante, es posible que en ciertas ocasiones, y en una misma zona de estudio, los diversos modelos de flujo operen de forma combinada (MOORE y FOSTER, 1990). Los modelos son más complementarios que contradictorios y pueden desarrollarse a escasos metros de distancia (DUNNE, 1978; MORISAWA, 1985). En una misma ladera mientras que en la zona superior y media de la misma la capacidad de infiltración es baja, en la parte inferior, en contacto con el fondo de valle, la capacidad de infiltración aumenta.

\section{ZONA DE ESTUDIO}

La cuenca hidrográfica de Guadalperalón se sitúa $22 \mathrm{Km}$ al noreste de la ciudad de Cáceres (Fig. 2), dentro del término municipal de Trujillo, y pertenece a la cuenca del río Magasca, incluida a su vez y de forma progresiva, en las cuencas de los ríos Tamuja, Almonte y Tajo. Tiene una superficie de 35,4 hectáreas y presenta forma alargada con orientación meridiana.

Se halla integrada en la mitad oriental de la unidad geomorfólogica de la Penillanura Cacereña (superficie de erosión finimiocena) (GÓMEZ AMELIA, 1985).

Los suelos se han desarrollado sobre un sustrato pizarroso (Complejo Esquisto Grauváquico) y se encuadran en el grupo de los Leptosoles y Regosoles según sus características topográficas y/o fisiográficas. La profundidad de los mismos oscila entre los 0 y $120 \mathrm{~cm}$, su porosidad y densidad son de 51,5\% y $1,26 \mathrm{gr} \mathrm{cm}^{-3}$ respectivamente, la textura es principalmente franca, la estructura granular y los valores de materia orgánica bajos (media del 2,7\%).

El clima es mediterráneo, con influencias tanto oceánicas como continentales, siendo los inviernos suaves y los veranos calurosos (la temperatura media anual es de $16^{\circ} \mathrm{C}$ ). La precipitación media anual es de $512 \mathrm{~mm}$ distribuidos en 85 días de lluvia. La variabilidad anual e interanual de las precipitaciones es alta, predominando e 1 número de años con valores inferiores a los medios. 


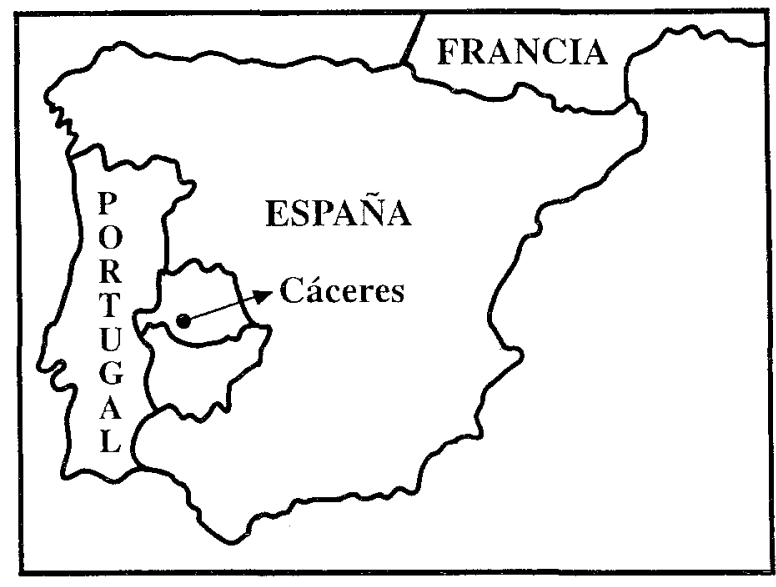

Figura 2.- Localización de la zona de estudio.

La vegetación potencial pertenece a la serie climatófila mesomediterránea luso-extremadurense de la encina (LADERO ÁLVAREZ, 1987). En Guadalperalón hay zonas arboladas de Quercus rotundifolia, con densidades de 15 a 40 pies por hectárea y zonas sin arbolado, donde domina Lazandula stoechas. Los fondos de valle de la cuenca rellenos de sedimento se hallan cubiertos por plantas herbáceas. El aprovechamiento principal de la dehesa de Guadalperalón es el ganadero, con una cabaña actual de 2,3 ovejas/Ha y 0,3 vacas/Ha.

\section{METODOLOGÍA}

Para medir las intensidades de cada evento de lluvia, se dispone de un pluviómetro tipo balancín modelo ARG100 (fabricado por Omnidata International LTD), con 0,2 mm de resolución, conectado a un registrador automático (fabricado por Unidata) que toma datos en intervalos de 5 minutos. A pocos metros del pluviómetro anterior hay instalado un pluviómetro totalizador.

La escorrentía de las vertientes se cuantifica por medio de 17 parcelas abiertas (trampas tipo Gerlach de 0,5 $\mathrm{m}$ de ancho) distribuidas en 5 unidades caracterizadas por su relación suelo-vegetación (SCHNABEL, 1997). Las muestras son recogidas inmediatamente después de cada suceso de precipitación y el total de la escorrentía es 
cuantificado con una probeta de 1 litro de capacidad. El valor de escorrentía utilizado para cada evento es el obtenido de la media de las 17 cajas Gerlach monitorizadas.

La cobertura del suelo se ha medido muestreando mensualmente los dos primeros metros cuadrados vertiente arriba de cada caja Gerlach mediante el uso de un bastidor con 100 puntos de contacto. Así se determina el porcentaje de cubrimiento de: suclo desnudo, hierba fresca, hierba seca, arbustos (Lavandula stoechas), afloramientos de roca madre, piedras sueltas, musgos y líquenes.

Cada 7-10 días se ha medido el porceritaje medio de humedad del suelo mediante el método gravimétrico (MARTÍNEZ-FERNÁNDEZ y LÓPEZ BERMÚDEZ, 1996).

\section{RESULTADOS Y DISCUSIÓN}

- Descripción de las precipitaciones

La precipitación total anual durante el año hidrológico 1994-95 ha sido de 331,5 mm, mientras que en 1995-96 la cifra se elevó a 720,1 mm distribuidos mensualmente según muestra la figura 3.

Según la propuesta metodológica del INSTITUTO NACIONAL DE METEOROLOGIA (1994), el año 1994-95 puede clasificarse como de "muy seco" y el año 1995-96 de "muy húmedo".

Las intensidades de la lluvia del primer año han estado por debajo del promedio, mientras que en el segundo año han estado por encima. En 1994-95 sólo el 9,7\% de los días de lluvia superó los $10 \mathrm{~mm}$ de precipitación, mientras que en 1995-96 lo hizo el $30,7 \%$, siendo $19,1 \%$ la media registrada en Cáceres para un periodo de 85 años.

La distribución estacional de precipitaciones e intensidades tampoco se $\mathrm{h}$ a ajustado a la media. Las abundantes lluvias caídas entre mediados de noviembre de 1995 y finales de enero de 1996 provocaron que las vertientes llegaran al tope de su capacidad de retención hídrica, alcanzando la capacidad de campo. 


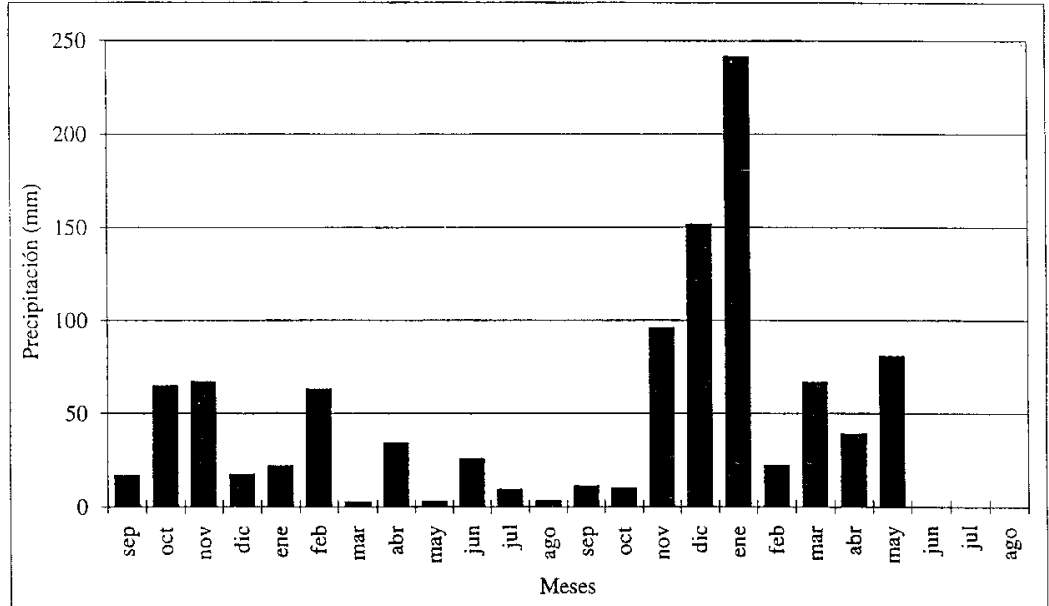

Figura 3.- Distribución mensual de la lluvia durante los años hidrológicos 1994-95 y 1995-96 en la cuenca de Guadalperalón.

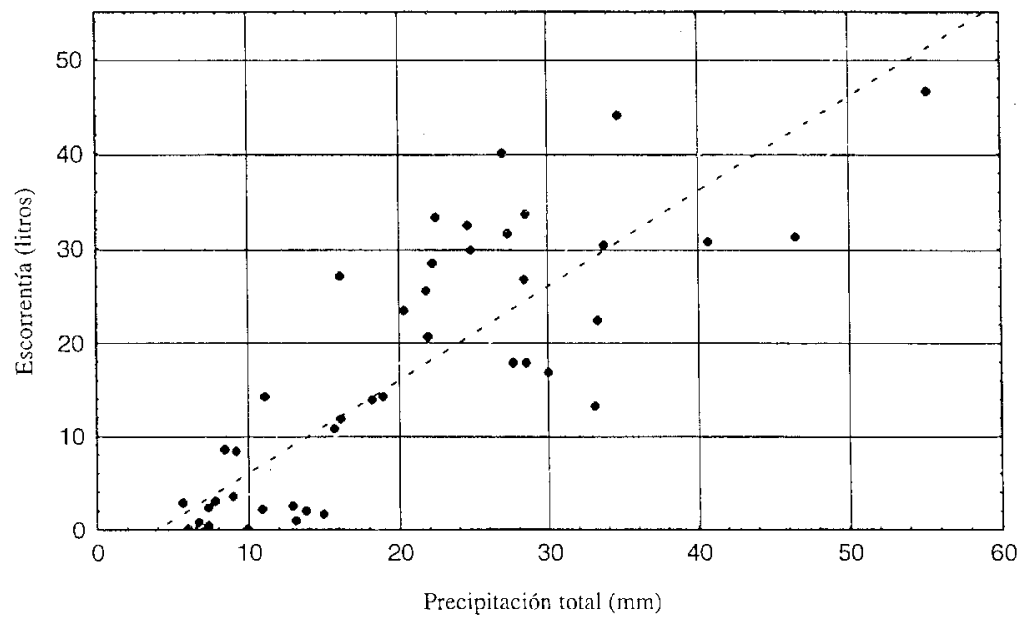

Figura 4.- Correlación lineal entre precipitación total y escorrentía media $\left(\mathrm{N}=48 ; \mathrm{r}^{2}=0,712\right.$; error estándar $=7,5 \mathrm{l} ; \mathrm{p}$-value $\left.=0,000\right)$. 


\section{- Determinación del umbral de escorrentía}

De los 42 eventos que han generado escorrentía en las vertientes durante el período de estudio, $5,7 \mathrm{~mm}$ ha sido el valor de precipitación total más bajo registrado. Sin embargo se han observado 6 eventos de lluvia con una precipitación comprendida entre 5,7 y $10 \mathrm{~mm}$, todos ellos con unas intensidades inferiores a $10 \mathrm{~m} 10 \mathrm{~mm} \mathrm{~h}^{-1}$ en 10 minutos (I-10) y $5 \mathrm{~mm} \mathrm{~h}^{-1}$ en 30 minutos (I-30), que no se han traducido en escorrentía. Estas circunstancias permiten establecer un umbral de escorrentía que estaría en $5,7 \mathrm{~mm}$ de precipitación total, con intensidades máximas en 10 y 30 minutos por encima de 10 $\mathrm{mm} \mathrm{h}^{-1}$ y $5 \mathrm{~mm} \mathrm{~h}^{-1}$ respectivamente.

El registro de un evento con valores inferiores al umbral establecido $(7,8 \mathrm{~mm}$ de precipitación total; $8,4 \mathrm{~mm} \mathrm{~h}^{-1}$ de I-10 y $3,0 \mathrm{~mm} \mathrm{~h}^{-1}$ de I-30) y con escorrentía superficial en las vertientes, pone de manifiesto la influencia de otros factores, además de la precipitación total e intensidades, en la determinación del umbral de escorrentía: desarrollo de una cubierta vegetal sobre el suelo, que intercepta el agua de la lluvia y altera la microtopografía del terreno, y la humedad edáfica que determina el tiempo de encharcamiento y la tasa de infiltración. En el caso del evento con valores por debajo del umbral de escorrentía establecido, el porcentaje medio de suelo desnudo en las vertientes era alto $(68,7 \%)$, así como el porcentaje de humedad $(18,8 \%)$. Con 1 a salvedad del evento anterior, para unas intensidades I-10 inferior a $10 \mathrm{~mm} \mathrm{~h}^{-1}$ e I-30 inferior a $5 \mathrm{~mm} \mathrm{~h}^{-1}$, es necesario una precipitación total de al menos $13 \mathrm{~mm}$ para generar escorrentía superficial en las vertientes.

\section{- Factores que explican la escorrentía superficial en vertientes}

En el análisis de las relaciones entre precipitación y escorrentía se han considerado los 48 eventos con valores de precipitación total por encima del umbral mínimo capaz de generar escorrentía, aunque 6 de ellos no la hayan producido. Relacionando ambas variables (escorrentía como variable dependiente y precipitación total como variable independiente), mediante la técnica estadística de la correlación lineal se obtiene un valor $\mathrm{r}^{2}$ de 0,712 ; que nos da un grado de explicación próximo al $75 \%$ de la variable dependiente.

Diversos estudios muestran que la mayor o menor permeabilidad de un suelo, y su capacidad o incapacidad para generar escorrentías superficiales rápidas, determina el grado de correlación entre precipitación total y escorrentía. En zonas con sustratos permeables y capacidad de retención hídrica elevada el nivel de correlación entre ambas variables no es bueno (LLORENS y ot., 1992; RUIZ FLAÑO, 1993), mientras que en áreas con poca capacidad de infiltración, como la cuenca de Guadalperalón, el grado de correlación es significativo (SALA, 1988; SALA y CALVO, 1990; GARCÍA y ot., 1993). 


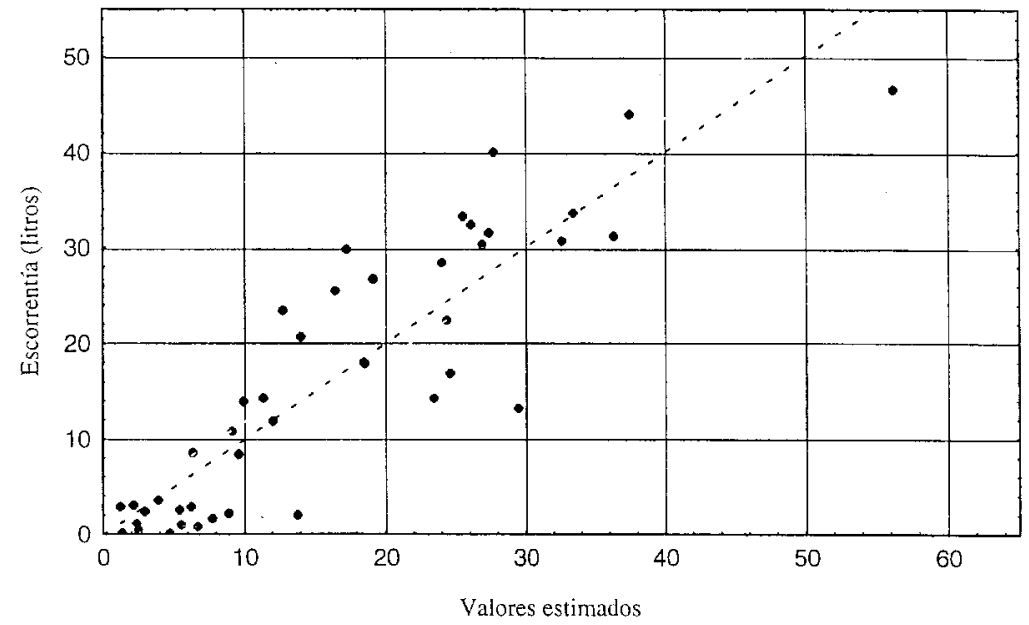

Figura 5.- Regresión múltiple entre precipitación total e I-10 (variables independientes) y escorrentía media (variable dependiente). Años hidrológicos 1994-95 y 1995-96 $\left(\mathrm{N}=48 ; \mathrm{r}^{2}=0,806\right.$; error estándar=6,3 1; $\mathrm{p}$-value=0,000).

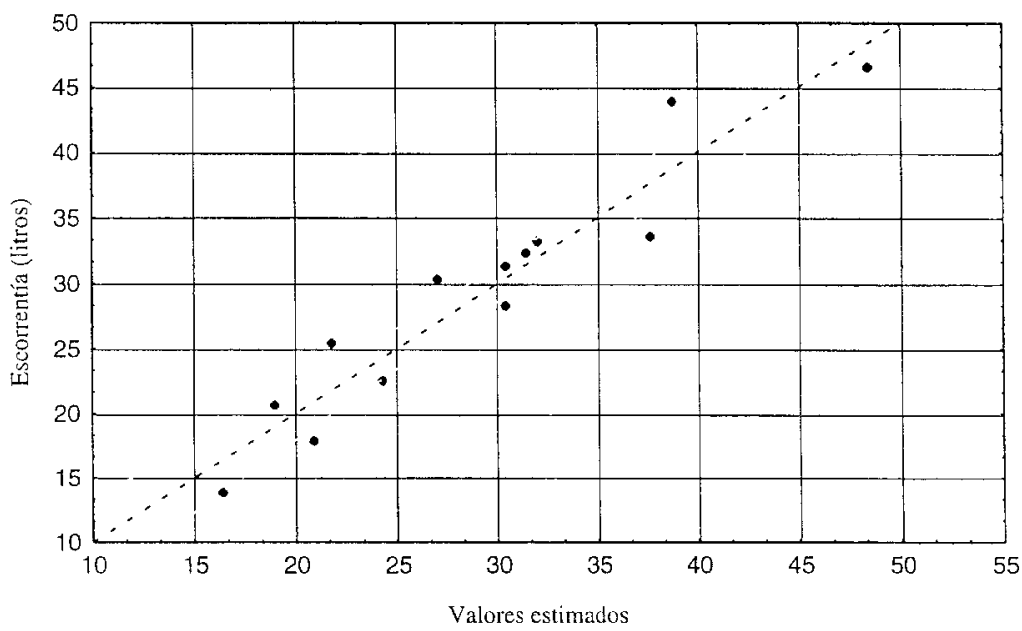

Figura 6.- Regresión múltiple entre precipitación total e I-10 (variables independientes) y escorrentía media (variable dependiente) durante el tiempo en que han estado saturados los suelos de la cuenca $\left(\mathrm{N}=13 ; \mathbf{r}^{2}=0,906\right.$; error estándar=3,2 l; p-value=0,000). 
A pesar del grado de correlación obtenido, la dispersión de la nube de puntos respecto a la recta de regresión (Fig. 4) indica la influencia de otros factores, como intensidad, distribución de la lluvia durante el evento, humedad antecedente del suelo, cubierta vegetal, microtopografía, etc, que modifican la influencia directa de la precipitación total sobre la escorrentía superficial.

Se han realizado dos conjuntos de correlaciones lineales entre intensidades y escorrentías medias, cuyos resultados aparecen en la tabla 1.

\begin{tabular}{|ccc|}
\hline Intensidades & Todos los eventos $(\mathrm{N}=48)$ & Capacidad de campo $(\mathrm{N}=13)$ \\
\hline I-10 & 0,348 & 0,570 \\
I-30 & 0,383 & 0,595 \\
I-60 & 0,448 & 0,616 \\
I-120 & 0,501 & 0,537 \\
I-240 & 0,570 & 0,468 \\
\hline
\end{tabular}

Tabla 1.- Coeficientes de correlación $\left(\mathrm{r}^{2}\right)$ correspondientes a las regresiones lineales entre diferentes intensidades de lluvia y la escorrentía media en vertientes. $\mathrm{I}-10=$ intensidad máxima en 10 minutos,..., I-240 = intensidad máxima en 240 minutos.

En general, el grado de correlación $\left(\mathrm{r}^{2}\right)$ obtenido no es bueno. Considerando todos los eventos, los valores más altos se obtienen en I-120 e I-240 debido a que una alta proporción de la precipitación total se concentra en períodos de tiempo comprendidos entre 2 y 4 horas. Sin embargo, cuando los suelos alcanzan su capacidad de campo la tendencia es la contraria.

Estos hechos demuestran la diversidad de factores que intervienen en la generación y producción de escorrentía superficial y las limitaciones que ofrece una técnica bivariante (regresión lineal) en la determinación del grado de explicación de cada factor. Aplicando la regresión múltiple se ha podido estudiar con mayor detalle el grado de interacción entre los diversos factores que explican la variable dependiente (JOHNSTON, 1980).

Tras múltiples combinaciones la correlación más alta $\left(\mathrm{r}^{2}=0,806\right)$ se ha obtenido relacionando precipitación total e I-10 (variables independientes) con escorrentía (variable dependiente), lo que demuestra una clara dependencia positiva de la producción de flujo de la cuantía e intensidad de las precipitaciones (GONZÁLEZ HIDALGO, 1992). No obstante, en la Figura 5 se aprecia como con escorrentías inferiores a 5 litros la explicación de la variable dependiente es compleja y existe una gran dispersión en la base. 
Aunque la introducción de los datos correspondientes a humedad edáfica y porcentaje de superficie de suelo desnudo no mejoran los resultados de la regresión anterior, aislando los 13 eventos de lluvia sucedidos en los meses de mayor pluviosidad y suelos con capacidad de campo, se obtiene un $\mathrm{r}^{2}$ de 0,906 ; con un ajuste de la nube de puntos a la recta muy grande (Fig. 6). El establecimiento de una tasa de infiltración constante muy baja explica el alto grado de correlación.

Otro factor que finalmente se ha considerado en el presente estudio ha sido la distribución de la lluvia durante el evento. Dependiendo de la localización de las intensidades máximas del evento durante el desarrollo del mismo, su efecto sobre la generación y producción de escorrentía variará de forma notable. La denominada "estructura temporal del evento" es considerada por algunos investigadores (OLLESCH y SEUFFERT, 1995) como el principal factor para explicar la escorrentía. La aplicación de un "índice de homogeneidad" $(P)$ para el estudio de la influencia de la "estructura temporal" de la lluvia sobre la escorrentía, apenas mejora el resultado obtenido en la regresión múltiple, considerando sólo la precipitación total y la $\mathrm{I}-10$, debido al alto grado de explicación de ésta (CEBALLOS et al., en prensa). No obstante, este factor sí es útil para explicar el comportamiento excepcional de algunos eventos muy puntuales.

Aunque el modelo de escorrentía más frecuente en la cuenca haya sido el descrito por HORTON (1940), durante períodos de lluvias continuadas se ha observado en muchas zonas de la cuenca flujo superficial de saturación (HEWLETT, 1961; DUNNE y BLACK, 1970) más flujo de retorno y flujo subsuperficial.

\section{CONCLUSIONES}

El umbral de precipitación necesario para generar escorrentía en las vertientes ha sido de 5,7 mm de precipitación total, con I-10 superior a $10 \mathrm{~mm}$ h-1 e I-30 superior a $5 \mathrm{~mm}$ h-1. No obstante, la cubierta vegetal y la humedad antecedente del suelo pueden condicionar el umbral establecido.

Los factores que mejor explican la generación y producción de escorrentía superficial en las vertientes son la precipitación total y la intensidad máxima en 10 minutos, con un $r^{2}$ de 0,806. La introducción de otros factores en el modelo de regresión, como porcentaje de suelo desnudo o porcentaje de humedad antecedente, no ha logrado incrementar el grado de explicación del mismo. No obstante, si se considera únicamente la serie de eventos con la cuenca próxima al estado de saturación, el ajuste del grado de la correlación aumenta considerablemente, alcanzándose un $\mathrm{r}^{2}$ de $0,906,10$ cual denota, a pesar de no existir una correlación estadística, la importancia de la humedad del 
suelo para explicar los procesos de escorrentía. La distribución de la lluvia durante el evento mejora ligeramente el grado de correlación de la regresión y esclarece el comportamiento excepcional de algunos sucesos de lluvia.

Debido a la variabilidad espacial de la zona y del propio régimen pluviométrico durante el período estudiado se han observado en la cuenca de Guadalperalón flujo tanto "hortoniano" como superficial de saturación.

\section{AGRADECIMIENTOS}

Este estudio ha sido financiado por la Comisión Interministerial de Ciencia y Tecnología, en el marco del proyecto AMB95/0986-CO2-02, y apoyado por la Junta de Extremadura, Dirección General de Enseñanzas Universitarias e Investigación.

\section{BIBLIOGRAFÍA}

ANDERSON, M.G. y BURT, T.P.(1990): Process studies in hillslope hydrology: an overview. En ANDERSON, M.G. y BURT, T.P. (Eds): Process studies in hillslope hydrology, 1-8. John Willey and sons. England.

BERNET, R., SCHNABEL, S. y GÓMEZ AMELIA, D. (1994): La cubierta, vegetal como factor de degradación en una zona de dehesa (Provincia de Cáceres). En ARNÁEZ, J.; GARCÍA RUIZ, J.M. y GOMEZ VILLAR, A. (Eds): Geomorfología en España. III Reunión de Geomorfología, 211225. S.E.G. Logroño.

CAMPOS PALACÍN, P. (1993): "Valores comerciales y ambientales de las dehesas españolas". Agricultura y Sociedad, 66, 9-41, MAPA. Madrid.

CEBALLOS, A. (1997): Balance de agua de una cuenca hidrográfica bajo explotación de dehesa en Extremadura. Tesis Doctoral Inédita. Area de Geografía Física. Dpto. de Geografía y O.T., Universidad de Extremadura.

CEBALLOS, A. y SCHNABEL, S. (1996): Runoff generation in a small catchment under silvopastoral landuse in SW Spain. Conference on ecolydrological processes in small basins. ERB. Strasbourg, France (en prensa).

CEBALLOS, A., SCHNABEL, S. y CERDÁ, A. (1996): "El efecto de la escala sobre los procesos de escorrentía superficial". En GRANDAL, A. y PAGES, J. (Eds): IV Reunión de Geomorfología, S.E.G. Cuadernos del Laboratorio Xeolóxico de Laxe. 21, 91-102. A Coruña.

CEBALLOS, A., FERNÁNDEZ-TOLEDO, P. y GÓMEZ-AMELIA, D. (en prensa): "Propuesta de un "indice de homogeneidad" para el estudio de la influencia de la "estructura temporal de la lluvia" sobre la escorrentía en zonas semiáridas". Investigaciones Geográficas. 18. Instituto Universitario de Geografía. Universidad de Alicante. 
DUNNE, T. (1978): Field studies of hillslope flow processes. En KIRKBY, M.J. (Ed): Hillslope hydrology. 227-293. John Willey and sons. Great Britain.

DUNNE, T. y BLACK, R.D. (1970): "Partial-area contribution to storm runoff in a small" New England watershed. Water Resources Research. 6, 478-490.

FREEZE, R.A. (1972): "Role of subsurface flow in generating surface runoff" Water Resources Research. 8(5), 1272-83.

GARCÍA, C., LLEAL, I., PEÑA, J.C. y JARDÍ, M. (1993): "Instalación de parcelas experimentales para el control de la arroyada en superficie: primeros resultados en una cuenca de Argentona". Notes de Geografia Física. 22, 113-120. Universidad de Barcelona.

GERITS,J., DE LIMA, J. y VAN DEN BROEK, T.M. (1990): Overland flow and erosion. En ANDERSON, M.G. y BURT, T.P. (Eds): Process studies in hillslope hidrology. 173-214. John Willey and sons. England.

GÓMEZ AMELIA, D. (1985): La penillanura cacereña. Estudio geomorfológico. Dpto de Geografía. Facultad de Filosofía y Letras. Servicio de Publicaciones. Universidad de Extremadura.

GÓMEZ AMELIA, D. y SCHNABEL, S. (1992): "Procesos sedimentológicos e hidrológicos en una pequeña cuenca bajo explotación de dehesa en Extremadura". En LOPEZ BERMÚDEZ, F.; CONESA GARCIA, C. y ROMERO DÍAZ, M.A. (Eds): II Remnión Nacional de Geomorfología. 55-63. S.E.G. Murcia.

GONZÁLEZ HIDALGO, J.C. (1992): Pantas espaciales de la erosión hídrica en el semiárido aragonés. Exposición topográfica y cubierta vegetal. Factores de erosión. Tesis Doctoral Inédita. Departamento de Geografía y O.T. Universidad de Zaragoza.

HEWLETT, J. D. (1961): Soil moisture as a source of base flow from steep mountain watersheds. U.S. Dep. Agric. Fo. Serv., Southeastern For. Exp. Stn., Asheville, N.C., Stn. Pap. No. 132.

HORTON, R.E. (1940): "An approach toward a physical interpretation of infiltration capacity". Proc.Soil Sci. Soc. Am., 5, 399-417.

I.N.M. (1994): Calendario Meteorológico 1993. Madrid.

JOHNSTON, R.J. (1980): Multivariate Statistical Analysis in Geograply. Longman. Great Britain.

LADERO ÁlVAREZ, M. (1987): La España Luso-Extremadurense. En PEINADO LORCA, M. y RIVAS-MARTÍNEZ, S. (Eds): La vegetación de España. 455-488. Colección Aula Abierta. Universidad de Alcalá.

LLORENS, P., LATRON, J. y GALLART, F. (1992): "Analysis of the role of agricultural abandoned terraces on the hydrology and sediment dynamics in a small mountainous basin. (Hight Llobregat, Eastern Pyrenees)". Pirineos. 139, 27-46. Jaca.

MARTÍNEZ FERNÁNDEZ, J. y LÓPEZ BERMÚDEZ, F. (1996): "Métodos para el estudio de las propiedades hídricas de suelos y formaciones superficiales". Cuademos Técnicos de la S.E.G. Geoforma Ediciones. Logroño. 
MOORE, I.D. y FOSTER, G.R.(1990): Hydraulics and overland flow. En ANDERSON, M.G. y BURT, T.P. (Eds): Process studies in hillslope hidrology. 215-254. John Willey and sons. England.

MORISAWA, M. (1985): Rivers. Form and Processes. Gomorplology Text. Edited by K.M. Clayton. Longman. London and New York.

OLLESCH, G. y SEUFFERT, O. (1995): The impact of rainfall variability on runoff and erosion in pasture ecosystems. En Conference on Erosion and Land Degradation in the Mediterranean. 115124. University of Aveiro. Portugal.

RUIZ FLAÑO, P. (1993): Procesos de crosión en campos abandonados del Pirmeo. Monografías científicas n"4. Geoforma Ediciones. Logroño.

SALA, M. (1988): "Slope runoff and sediment production in two mediterranean mountain environments". Catena Supplentent. 12, 13-29. Braunschweig.

SALA, M. y CALVO, A. (1990): Response of four different mediterranean vegetation types to runoff and erosion. En THORNES, J.B. (Eds): Vegetation and Erosion. Processes and Environments. 347-362. John Willey and sons. England.

SCHNABEL, S. (1997): Soil erosion and runoff production in a snall watershed under silvo-pastoral landuse (dehesas) in Extrenadura, Spain. Geoforma Ediciones. Logroño.

SCHNABEL, S. y GÓMEZ AMELIA, D. (1993): Temporal variability of soil loss in areas under sil-pastoral landuse in Central Spain. En Workshop on Soil Erosion in Semi-Arid Mediterranean Areas. 265-273. Taormina. Italy.

SCHNABEL, S., GÓMEZ AMELIA, D. y BERNET, R. (1996): "La pérdida de suelo y su relación con la cubierta vegetal en una zona de dehesa". En CAMPESINO, A. y VELASCO, C. (Eds): VII Coloquio Ibérico de Geografin. 195-206. A.G.E.. Dpto de Geografía y O.T. (UEX). Cáceres. 